\title{
APPLICATION OF ARTIFICIAL NEURAL NETWORK FOR FATIGUE ANALYSIS IN WIND TURBINE BLADE
}

\section{WILSON J. VELOZ PARRA, HAO BAI, YOUNES AOUES AND DIDIER LEMOSSE}

\author{
${ }^{1}$ Normandy University, INSA de Rouen, Mechanical Laboratory of Normandy, \\ 685 Avenue de l'Université, 76800 Saint-Étienne-du-Rouvray, France , \\ wilson.veloz_parra@insa-rouen.fr
}

Key words: Deep Learning Application, Multi-axial Fatigue, Wind Turbine Blade, Load Application Method

\begin{abstract}
Wind turbine blades are subjected to wind pressure distribution that depends on the external environment and the inertial loads from their rotational velocity, acceleration, and turbine control. To simulate these effects, numerical tools are used. Allowing a coupled nonlinear aero-hydro-servo-elastic simulation in the time domain representing the multibody 1D beam finite element model (FEM). Nevertheless, when the mechanical analysis comes into detail, a shell FEM with applied loads in 3D spatial space must be used to analyze the fatigue. Therefore, the loads estimated by beam simulations need to be transferred into an equivalent 3D distributed loads for the shell FEM Called in the literature Load Application Methods (LAM), Each of these LAM differs in the stress distribution and the deflection of the blade. Subsequently, fatigue analysis of the whole blade can be performed by defining the cycle counting method and multi-axial damage criteria for composite material. However, this process is computationally expensive, since it is required to calculate the stress history in the shell FEM of the blade for each time instant of the aero-elastic simulation for different mean wind speed, other authors use a damage equivalent load (DEL) to estimate the fatigue damage directly from the 1D simulations. To reduce the number of call of the aero-elastic and shell FEM simulation a deep neural network (DNN) was trained to predict the accumulated fatigue damage in a node of the blade given the 10 minutes mean wind speed and the empirical cumulative density function of the damage per cycles with a relative error less than 5\%
\end{abstract}

\section{INTRODUCTION}

The wind turbine has developed over the last 35 years. They are more cost-effective and more reliable. The evolutionary period is not over, because the trending growth in power $(\mathrm{kW})$ continues in process, designing larger and more generation power wind turbine as the $10 \mathrm{MW}$ wind turbine developed by DTU [1] in 2013. Typically, numerical tools are used to facilitate the design of wind turbine blades, usually loads calculation is carried out with a beam Finite Element Model (FEM), taking into account aero-elastic behavior and turbine control commands. One example of these codes are the Fatigue, Aerodynamics, Structures, and Turbulence (FAST)[2] from National Renewable Energy Laboratory.

These multi-physics and multi-body aero-servo-hydro-elastic beam finite element codes can run a great number of the design situations described by certification bodies [3], taking into account all different extreme loads acting in the structure, generating the loading history used to basic or detailed analysis. 
For a detailed analysis as buckling or fatigue analysis, is necessarily a high-fidelity FEM representation of the wind turbine, but using usually this model requires a large amount of CPU time calculation and it's really difficult to incorporate the effects of the controller, energy production, and others behaviors as the beam aero-elastic simulations. To solve this problem, some authors have tried different approaches to transfer the time history loads from the beam simulations to a 3D load distribution to apply to a shell FEM, called in this article load application methods (LAM). Caous [4] has classified the methods reviewed in the literature into 4 groups. They aim to replicate the same behavior as the one produced by the $1 \mathrm{D}$ beam simulations. To estimate the fatigue damage in the wind turbine blade, different authors have to develop their methodologies to analyze the fatigue damage of wind turbines without using surrogate models. Some examples are the research presented by Dimitrov [5] defined a constant uniform pressure distribution as a linear function of the flap-wise bending moment and applied to the 3D FEM of a composite material wind turbine blade. Then, using the Hashin criterion calculates the ultimate failure of a composite laminate. The author used a progressive failure analysis, following the development of matrix-related failure events, until the first fiber failure occurs. for the fatigue failure for laminates a fatigue analog to the static Hashin failure criterion [6] with a progressive update of stiffness in the laminates. Subsequently, treatment of uncertainty in different assessments as uncertainties in wind climate variables in estimating extreme and fatigue loads, in the ultimate limit state, and fatigue limit state. And Also, the research by Bottasso [7] in the structural composite blade optimization. Divided into two major parts, first, optimizing a 2D FEM section and beam modeling, and then a 3D FEM is used to update the constraints of the 2D FEM optimization.
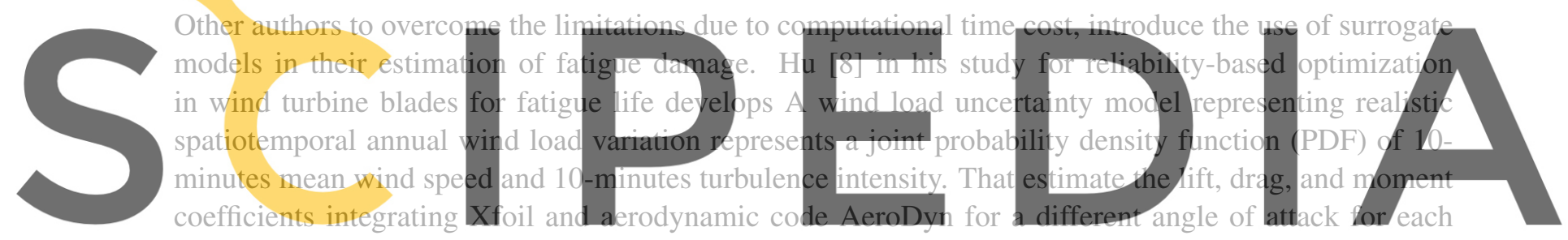

blade section. Then, applying a pressure distribution along the 3D FEM model of the blade to calculate

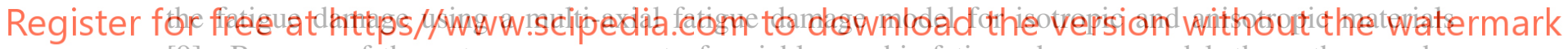

[9]. Because of the outrageous amount of variables on his fatigue damage model, the author used a

Kriging surrogate model [10] on defined hotspots, to estimate the reliability-based optimization of the wind turbine blade.

In a more recent study about surrogate models in wind turbine reliability assessment [11], the authors simulate the wind turbine using FAST code [2] to get resultant loads in a beam FEM of the wind turbine. Subsequently, Uses DELs implicitly assuming that a linear S-N curve can model fatigue strength of materials. The Miner's rule [12] is used to accumulate fatigue damage. Subsequently, a surrogate model using two different approaches are used to predict the damage at a different location of the blade, Kriging [13] and PCE (Polynomial Chaos Expansion) [14]. Then, making quantification of the surrogate model's uncertainty for different conditions on the wind turbine blade outputs. Modeling the wind turbine blade using beam FEM, the fatigue damage analysis is faster than using a 3D FEM. However, there is no detailed information on stress distribution along with the wind turbine, especially the blade which has the most complex geometry, and estimating this distribution is difficult to calculate. These methods mentioned before requires an enormous amount of simulations to train the surrogate model to estimate the fatigue damage, and this cost increases performing a $3 \mathrm{D}$ finite element analysis to estimate the fatigue 
damage over the wind turbine blade model.

These methodologies tend to use a large number of aero-elastic simulations for each combination of environmental conditions, in the study of Slot et al. [11] the number of aero-elastic simulations is 625 environmental cases and each of them is simulated 100 times to capture the time variation response. Using these aero-elastic time histories load responses to estimate the fatigue damage in a 3D finite element wind turbine blade model is an unattainable task because of the high computational cost of the 3D model. This study proposed a method to reduce the number of aero-elastic simulations and estimate the probabilistic fatigue damage using a 2D shell finite element wind turbine composite blade model. It selects first the environmental conditions for the 10 minute mean wind speed. Then 10 minutes aero-elasticservo simulations are performed at integer values between cut-in and cut-out wind speeds, subsequently, the load responses are filtered using Fourier transformation to reduce the time step of the load response. Then each time step of this filtered response is transferred into a 2D shell finite model of the composite blade using a load application method from the literature to estimate the detailed fatigue damage on the blade composites layers. To determine the fatigue damage is used multi-axial fatigue criteria based on a Tsai-hill criterion, constant life diagrams to estimate the fatigue strength at different stress ratios and Miner's rule for the fatigue accumulation. Once the fatigue damage is calculated a surrogate model based on deep neural networks is trained to predict the 10 minutes fatigue damage, taking as input parameters the 10 minutes mean wind speed and a uniform random variable that represents the empirical cumulative distribution function of the damage caused at each cycle.

A deep neural network is trained and validated to substitute this computational process taking as input variables the 10 minute mean wind speed and the empirical cumula
per cycle of the blade rot to predict the damage for a different time
with an error less than $5 \%$.
2 FATIGUE SIMULATION METHODOLOGY

2.1 Aero-elastic simulation
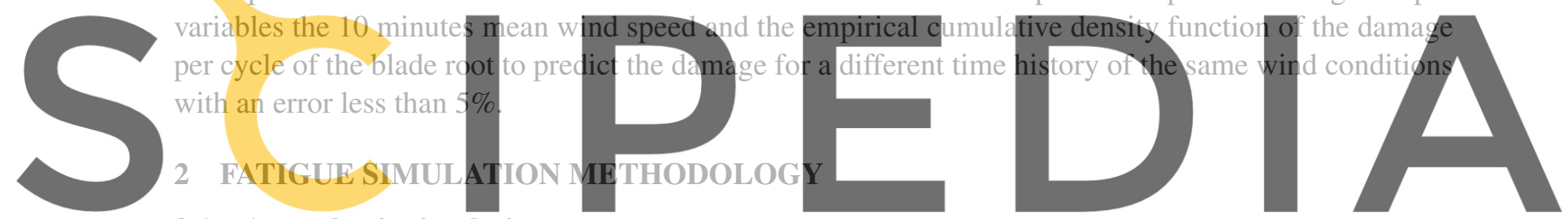

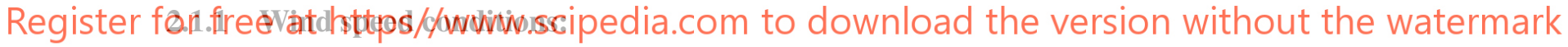

Measurements of wind speed during the last three years where extracted from open-data Engie Renewable project at la haute borne, Grand Est, France [15] collected for a 2MW wind turbine at a hub height of $80 \mathrm{~m}$. The wind turbine used in this study has a hub height of $119 \mathrm{~m}$ and cut-in and cut-out wind speeds of $[4,25] \mathrm{m} / \mathrm{s}$ respectively. In order to use these measurements, the wind speed is transformed to DTU hub height using a power law [16] and Hellmann's exponent coefficient $q=0.27$ for a condition of unstable air above human inhabited areas [17]:

$$
\bar{u}(z)=\bar{u}\left(z_{r e f}\right)\left(\frac{z}{z_{r e f}}\right)^{q}
$$

\subsubsection{Aero-elastic unsteady simulation:}

The IEC 61400-1 [3] define the dependency between wind speed (WS) and the standard deviation of the wind speed $\left(\sigma_{1}\right)$ in the Normal Turbulence Model. In this study case, a reference ambient turbulence intensity of a site Class 1A: $T I_{r e f}=0.16$. This dependency is given by the local statistical moments of 
$\sigma_{1}$ as: $\mathbf{E}\left(\sigma_{1} \mid W S\right)=T I_{\text {ref }}(0.75 W S+3.8)$ and $\mathbf{V}\left(\sigma_{1} \mid W S\right)=\left(1.4 T I_{r e f}\right)^{2}$. The details of the correlation parameter can be found in reference [3]. The correlation between $W S$ and mean shear exponent $\alpha$ is based on the simplified joint distribution defined by Dimitrov et al. [18].

Table 1: Wind turbine surrogate model inputs parameters.

\begin{tabular}{lcc}
\hline Input Parameter & PDF & PDF Parameter \\
\hline 10-min mean hub height wind speed & Weibull & $\mathbf{E}(W S)=7.28 \mathrm{~m} / \mathrm{s}$ \\
Std. of the wind speed during the 10-min simulation & Log-normal & $\mu_{\sigma_{1}}(W S), \sigma_{\sigma_{1}}(W S)$ \\
10-min mean shear exponent & Normal & $\mu_{\alpha}(W S), \sigma_{\alpha}(W S)$ \\
\hline
\end{tabular}

In this case, for the fatigue analysis, only uncertainties on the wind loads are considered. Other studies [11] take into account variation in multiple variables of the wind condition as mean wind speed, the standard deviation of the wind speed, shear exponent, wind direction, miss-align angle. This study only considers uncertainties in the 10 minutes mean wind speed to decrease the complexity of the problem and also reduce the sample size of simulations, decreasing the computational time. This simplification could lead to an underestimation of the fatigue damage of the wind turbine, however, it is considered as an initial step to evaluate and validate the proposed methodology. For the generation of the 3D wind field, the turbulence intensity and the shear exponent are constant values defined in the IEC standard [19]
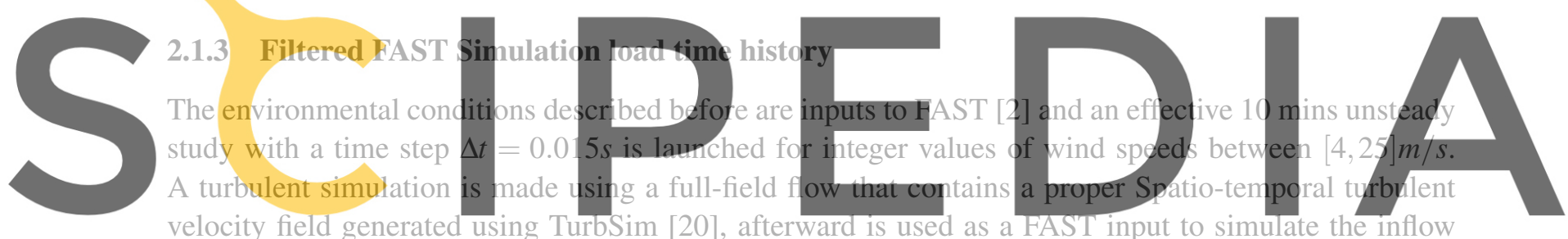
velocity field generated using TurbSim [20], afterward is used as a FAST input to simulate the inflow turbulent environment that incorporates many of the important fluid dynamic features.known to adversely

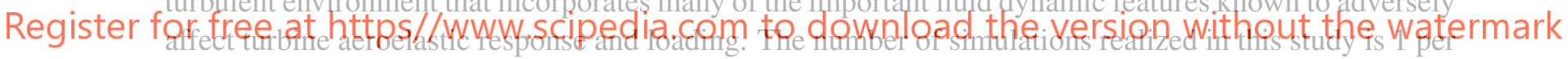
integer wind speed in the range $[4,25] \mathrm{m} / \mathrm{s}$ resulting in 21 FAST simulations.

Subsequently, a time history response of the forces and moments acting on 10 nodes of the blade are extracted as outputs from the FAST model resulting in 60 outputs variables described as $F_{i}^{\text {Fast }}$ and $M_{i}^{\text {Fast }}$, where $i=x, y, z$ for each node. After 300 simulated seconds, these loads are re-sampled to reduce the time step to $\Delta t=0.63 \mathrm{~s}$ of the time history resulting for each wind speed and apply each time instant to the 2D FEM blade to create a high fidelity response resulting in a total of 21,000 finite element analysis.

The filter used to re-sample the aero-elastic response is based on the Discrete Fourier Transformation (DFT) [21, 22]. The DFT is the equivalent of the continuous Fourier Transformation (FT) for signals only knowing at $\mathrm{N}$ instants separated by sample times $\mathrm{T}$, a finite number of data points.

This method is used to down-sample the original signal by assuming is periodical, changing the sample space from $d t$ to $d t \cdot$ length $(T) / M$, where $\mathrm{M}$ is the new sample number. After re-sampling the loads, there is little reduction in the maximum and minimum value of the output. However, this reduction is not important for the magnitude of the loads and is used for further analysis. 


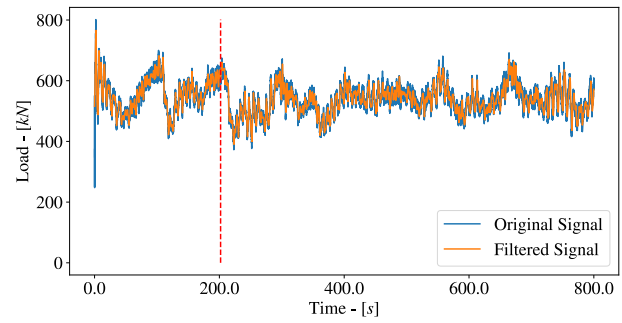

a)

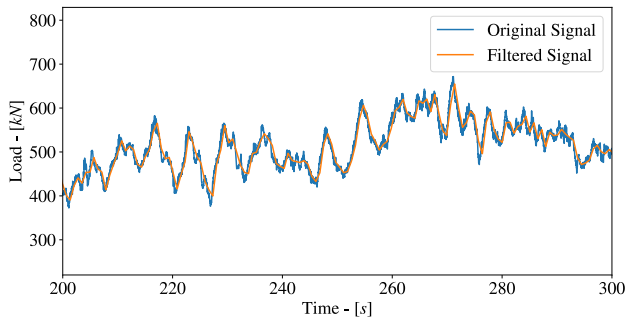

b)

Figure 1: FAST load output time history for $S p n 1 F L x b 1$ at $11 \mathrm{~m} / \mathrm{s}$ with a time step of $0.015 \mathrm{~s}$ and re-sampled to a time step of $0.63 s$, and a zoom between 200 and 300 seconds.

\subsection{Load application method for shell finite element model}

As the loads are extracted from an aero-elastic simulation code, the blade is modeled using 1D beam FEM, and these loads are the resulting load in a node. Simple analysis can be carried out as maximum load, frequency, and/or tip displacement analysis, however, if a more complex analysis, like fatigue or buckling analysis is required, it must use a 3D model of the blade with distributed loads.

To transfer the 1D load distribution from the beam FEM to a 3D load distribution to apply to a shell FEM, Caous has classified the methods reviewed in the literature into 4 groups: Application of loads by sections, application of loads by sections but physical distribution on sections, continuous application on the blade of an oriented surface load and Dis
application of an acceleration field and pressure
chosen a LAM from the second group applying
simplified physical laws.
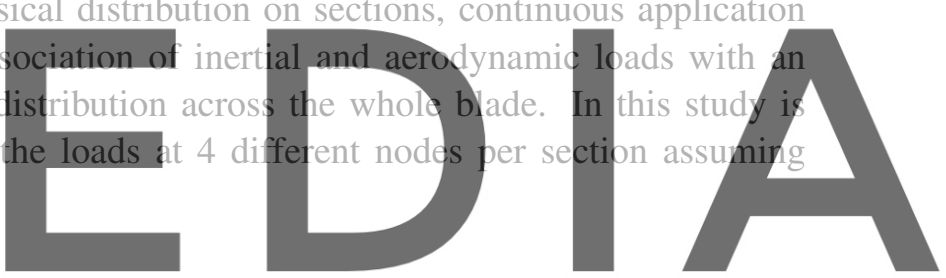

2.2.1 Blade Description

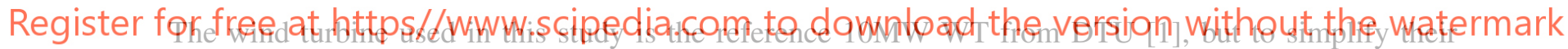
complex material distribution, the composite materials were changed to QQ1 and P2B from composite datauase SNL/DOE/MSU [23] and Balsa was retained as core material. The spatial material distribution is simplified, where, only 3 sections are defined in the span-wise direction and the transversal section is divided into 3 groups: Shear Webs (SW), Trailing Panel (TP), and Leading Panel (LP), each group has the same layup and thickness. Also, all layups are considered symmetric, reducing the number of thickness variables to 24 (9 thicknesses for TP, 9 for LP, and 6 for SW).

\subsubsection{LAM: 4 Nodes}

This approach distributes the forces and moments resultants between four nodes of each section, assuming simplified physical laws. Loads are applied by section and distributed across the section in such a way that sections stay deformable. They distribute external forces and moments extracted from the 1D beam FAST simulations on four points of the corresponding shell FEM sections. As explained by Caous [4], it describes six equations to distribute the loads and moments at the four nodes in each section. To know the details of this method read the article [4]. 


\subsection{Blade root fatigue evaluation}

Once the loads are applied to the 2D shell FEM, Python scripts are developed to extract the resulting stress components. After applying the loads time history in the 2D shell FEM using LAM 4 nodes, can be extracted the stress at each node and each position of every ply, these results in a total of more than 450,000 output values per time instant, in this case, to reduce the size of the output domain is only analyzed the inferior position of the inner ply of a node at the blade root to extract their stress time history in $\sigma_{x x}, \sigma y y$ and $\sigma_{x y}$ that will be used to calculate the multi-axial fatigue damage.

The stress distributions through a laminate have a piece-wise linear function, which is consistent with the classical laminate theory of composite materials.

\subsubsection{Multi-Axial Fatigue Criteria}

The stress time history got shows that the blade bears non-proportional multi-axial complex stress states of variable amplitude and mean, which is the same result obtained by [24]. Stress history of longitudinal normal stress $\sigma_{x x}$, transverse normal stress $\sigma y y$ and shear stress $\sigma_{x y}$ of blade root node 606 , are estimated only in QQ1 layer as this is the layer with the highest stress value.

To count cycles for the non-proportional multi-axial complex stress states, 800 seconds of the FAST simulation are executed and after 200 seconds, the remaining 600 seconds (10 min of an effective simulation) are used to count peak, valley, for each of the stress components. A range-mean counting method described by ASTM Committee [25] is applied to count all the half cycles, allowing a cycle-by-cycle
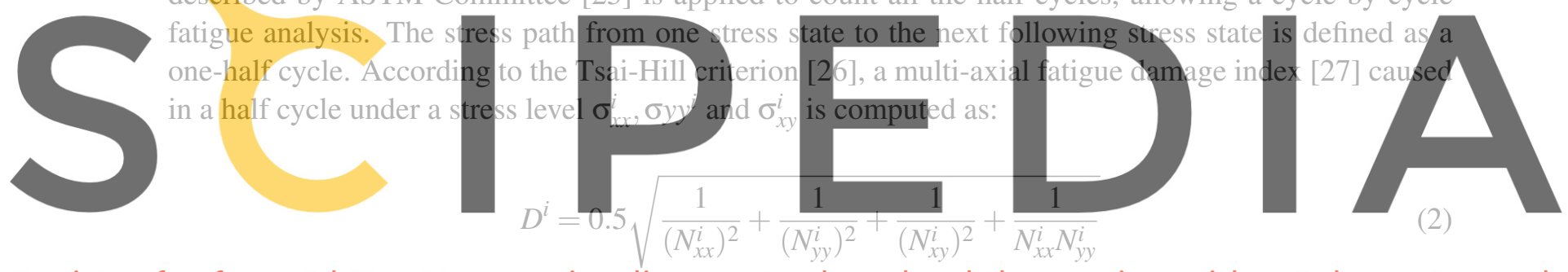

(2)

Register for free at https//www.scipedia.com to download the version without the watermark where $N_{x x}^{i}, N_{y y}^{i}$ and $N_{x y}^{i}$ are the number of allowable cycles under pure stress components $\sigma_{x x}^{i}, \sigma y y^{i}$ and $\sigma_{x y}^{i}$, respectively. The coefficient 0.5 indicates the half cycle.

\subsubsection{Fatigue damage accumulation}

To continue the fatigue analysis is necessary to select the estimation of the fatigue damage accumulation, which is nonlinear under variable amplitude non-proportional multi-axial loading. A comparative study of nonlinear damage accumulation in stochastic fatigue FRP (fiber-reinforced plastics) laminates by Sarkani [28] presents different non-linear methods versus a linear method and all of them predicted fatigue lives comparable to those predicted by the linear damage accumulation rule.

The Miner's rule [12], the most used method to predict fatigue damage of wind turbine blades, assumes a linear damage accumulation [3]. The fatigue damage in 10 minutes simulation is calculated as:

$$
D_{10}=\sum_{i=1}^{n} D^{i}=0.5 \sum_{i=1}^{n} \sqrt{\frac{1}{\left(N_{x x}^{i}\right)^{2}}+\frac{1}{\left(N_{y y}^{i}\right)^{2}}+\frac{1}{\left(N_{x y}^{i}\right)^{2}}+\frac{1}{N_{x x}^{i} N_{y y}^{i}}}
$$




\subsubsection{Constant life diagram}

The S-N curves or Wöhler diagram [29] are used to represent the fatigue properties of structures in terms of a damage accumulation law. In this representation, the number of cycles to failure is given as a function of the stress cycle's amplitude. In its typical form, the S-N fatigue life model [30] is provided by the relation:

$$
N_{i j}=a_{i j} S_{i j}^{b_{i j}}
$$

where $S_{i j}$ is the stress amplitude, $N_{i j}$ is the number of cycles to failure, $a_{i j}$ and $b_{i j}$ are fatigue strength coefficients corresponding to a stress ratio R. ASTM standards [30] suggest treating statistically the fatigue strength coefficient used to construct the S-N curve to represent a 95\% survival probability with a confidence interval of $95 \%$. However, S-N curves only capture the failure behavior at a constant ratio $R$, which is used for constant amplitude stress. In this case, the stress amplitude is variable through time, meaning that failure cycles at different ratios must be estimated. Here, is used a constant life diagram [31].

The constant life diagram (CLD) is a model used to predict the number of cycles to failure as a function of cycle amplitude $S$ and mean stress values by interpolating between S-N curves obtained from constant amplitude with different load ratios. CLD for composite materials QQ1 and P2B for longitudinal and transversal direction are constructed using fatigue test data extracted from SNL/MSU/DOE composite material database [23]. This test includes maximum stress, minimum stress, and cycles to failure for each material tested at ratios $R=[-2,-1,-0.5,0.1,0.5,0.7,10]$.
The constant life diagram is constructed from the $95 \%$ lower bound S-N curves. Vassilopoulos
studied the influence of the CLD formulation and concluded after analyzing different approaches that
piece-wise linear is the most accurate of the approaches when using a reasonable number of S-N curves
$(>3)$. The piece-wise linear CLDs of QQ1 and P2 B has a range of eycles to lailure that goes fom $10^{2}$
to $10^{8}$.

Register for free at https//www.scipedia.com to download the version without the watermark 2.4. Surrogate model: regression deep neural network

The surrogate model selected for this probiem is the deep neural network (DNN) due to the increasing development of this technology and problem complexity. DNN is an artificial neural network with multiple layers between the input and output layers that can model complex functions [33].

\subsection{1 input domain}

The input parameters to generate the environmental conditions are the 10 minutes mean wind speed, 10 minutes turbulence intensity, and 10 minutes mean shear exponent. Only one simulation per wind speed between $(4,25) \mathrm{m} / \mathrm{s}$ was performed, resulting in a total of 21 stress time history used to calculate the damage caused in a half cycle $D^{i}$ per wind speed $W S$, for a unique value of $W S$ multiple values of $D^{i}$ are correlated as shown in figure 2(a). As can be seen, if a neural network is used to predict the damage, it only predicts a mean value per wind speed, resulting in a bad prediction of $D^{i}$. To predict the 10 min damage $D_{10}$ is added as the second input parameter, the empirical cumulative distribution function (ECDF) of $D^{i}$ per each wind speed ordered as an increasing function, using python package statsmodel 
[34]. Adding this second input parameter, the damage prediction for $D_{10}$ is estimated by selecting the desired wind speed and generating a uniform distribution function $[0,1]$, of size equal to the number of half-cycles in a 10 min stress history (i.e., count of ranges of stress).
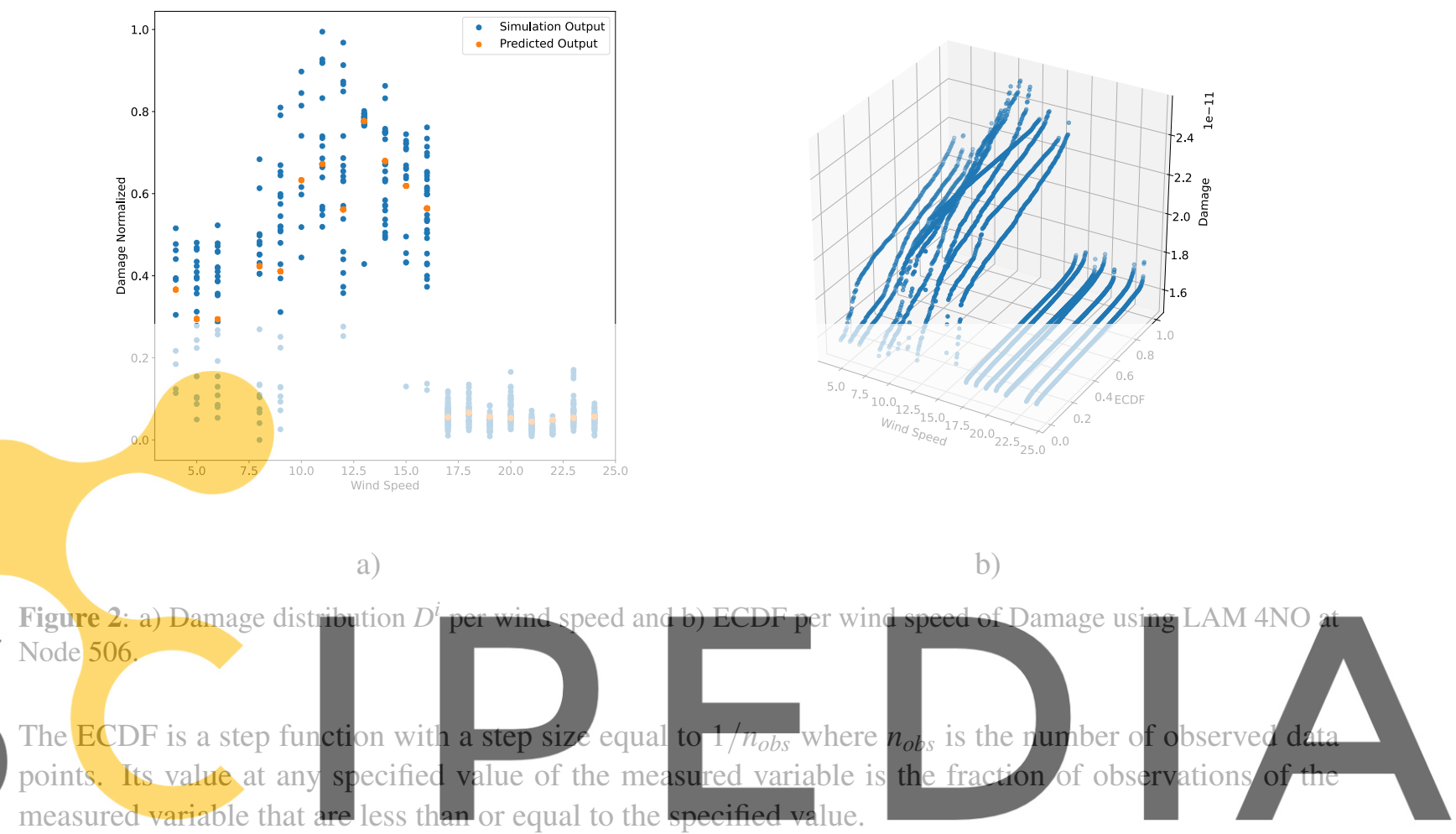

measured variable that are less than or equal to the specified value.
The use of ECDF in this problem is motivated by the idea of the inverse transform method [35], the

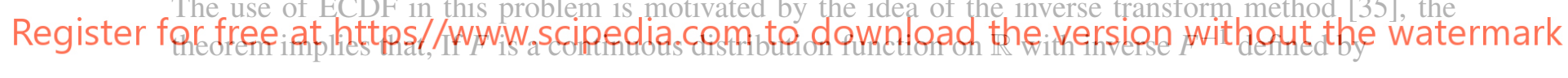

$$
F^{-1}(u)=\inf x: F(x)=u, 0<u<1
$$

If $U$ is a uniform $[0,1]$ random variable, then $F^{-} 1(U)$ has distribution function $F$. Also, if $X$ has distribution function $F$, then $F(X)$ is uniformly distributed on $[0,1]$. This theorem can be used to generate random variables with an arbitrary continuous distribution function $F$ provided that $F^{-1}$ is explicitly known.

As shown in figure 2 sorting the damage from lowest to highest, each value of $D_{i}$ per wind speed has a corresponding value of $\bar{F}$, which gives the network a better representation of the behavior of the damage.

\subsubsection{DNN description}

For training the DNN, all inputs and output parameters were scaled between $(0,1)$ to normalize all parameters magnitude, after the data is shuffled, $90 \%$ of the data was used to train the model and the left $10 \%$ for validating the model prediction. The DNN is a sequential fully connected network, having a total of 6 
hidden layers, each has 296 neurons, also all layers have a rectified linear unit (Relu) activation function and a learning rate of 0.001. For this regression problem, the weight and bias for the DNN are optimized using Adam optimizer to minimize the mean squared error of the difference between prediction and real value.

After the training and validation phase of the DNN, it can be used to predict the 10 minutes fatigue damage. The stopping criteria were a maximum iteration of 3000 epochs or an absolute error of validation mean squared error between two subsequent epochs.

\section{RESULTS}

\subsection{DNN prediction}

The training phase of the DNN are shown in figure 3 (a) the correlation between the damage simulated $D_{i}$ and the damage predicted $\hat{D}_{i}$ performed in the validation set is good, having an RMSE of $4.16 e-05$. Also, the relative percent error of each damage prediction is shown in figure 3 (b), the maximum relative error is about $3.5 \%$ which shows good accuracy of the DNN.

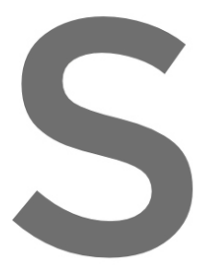

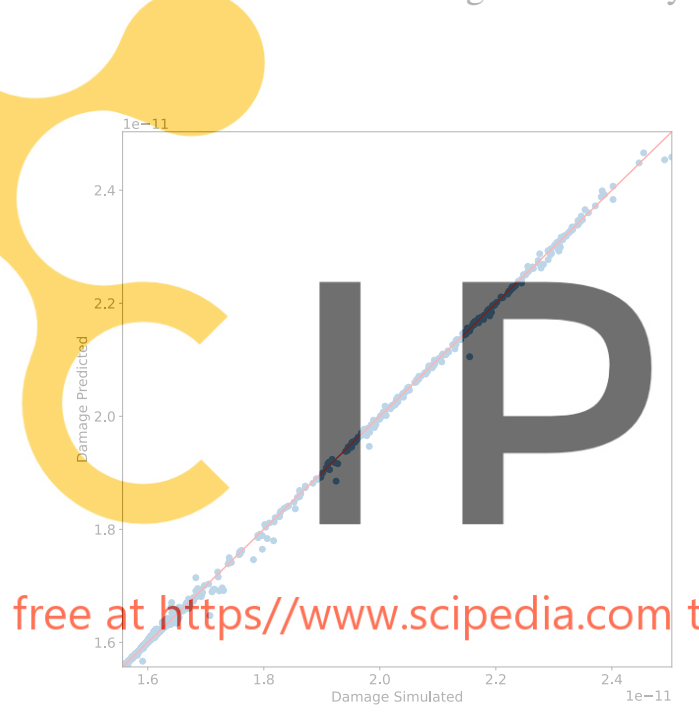

a)

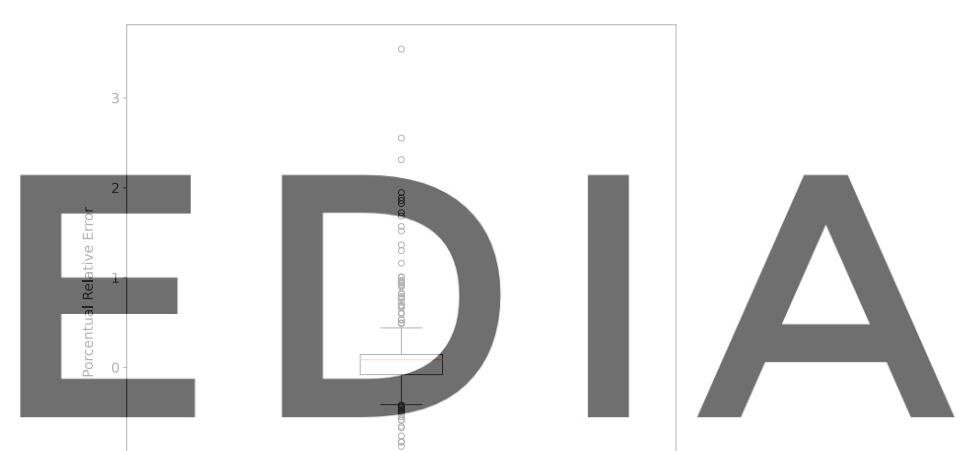

o download the version without the watermark

b)

Figure 3: a) correlation between damage simulated and damage predicted by DNN, b) prediction relative percent error.

But, these predictions are made over one aero-elastic simulation at 1 selected seed number, to test the predictions of 10 minutes damage $\hat{D}_{10}$ of the network a new seed number must be used to calculate the real accuracy of the trained network.

The 3D wind field is generated using a different seed number selected randomly and used to run the aeroelastic simulation to generate the time history of the behavior of the wind turbine blade, that subsequently was transfer to the blade shell FEM and calculated the 10 minutes damage at the blade root per each wind speed as shown in table 2. These $D_{10}^{S E E D=2}$ are used to test the response of the trained DNN to predict $\hat{D}_{10}$. The maximum relative percent error was found at wind speed $12 \mathrm{~m} / \mathrm{s}$ less than $5 \%$, meaning that 
Table 2: 10 minutes damage prediction testing using a different seed number for generation of the 3D wind field time history.

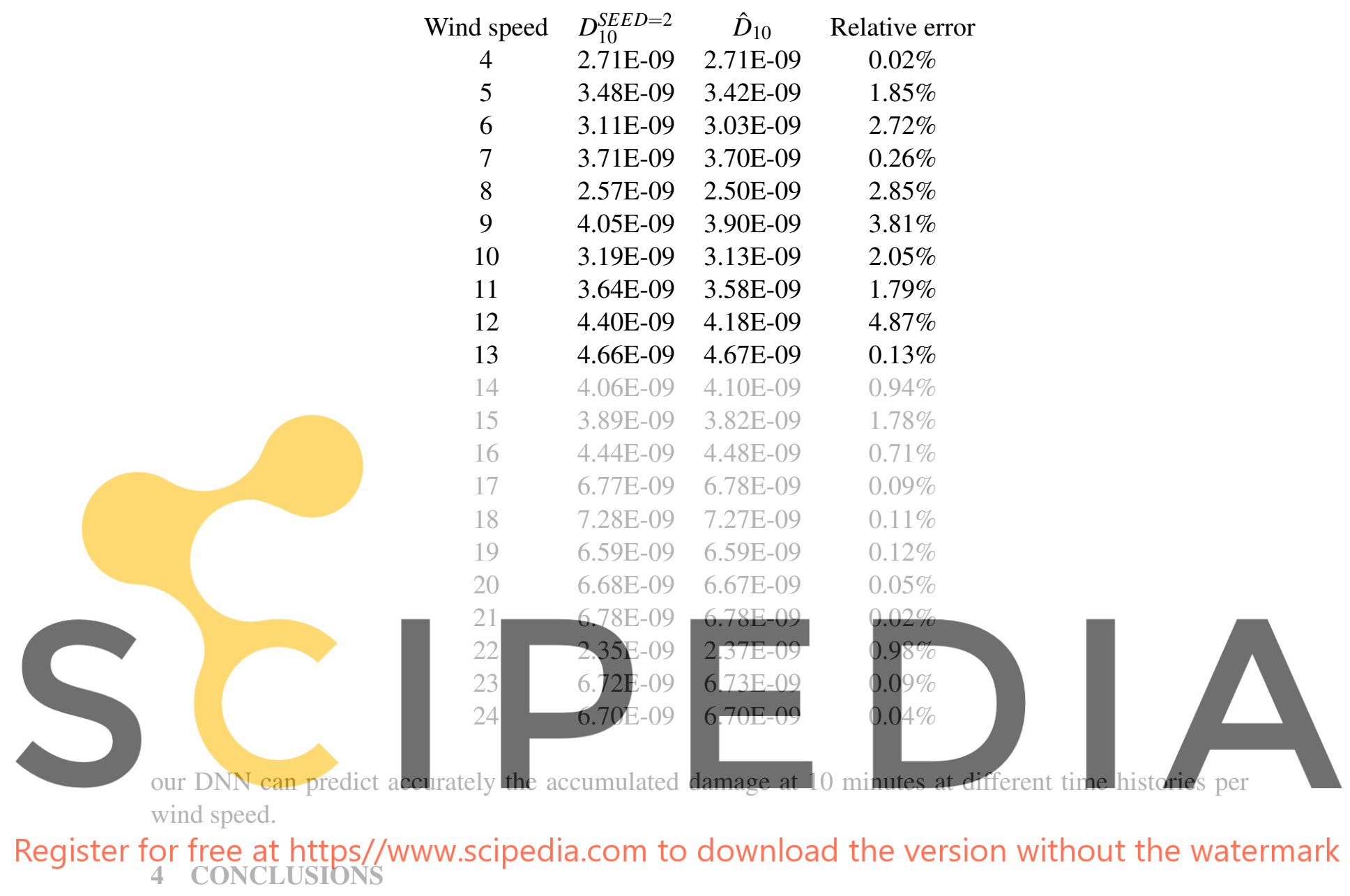

A 10 minutes fatigue analysis of a composite wind turbine blade is presented in this study. To reduce the calculation time is introduced a deep neural network (DNN), which takes as input values the wind speed and the empirical cumulative distribution function of the damage per cycle for 10 minutes, which can predict the accumulated 10 minutes of damage at one node of the blade root.

This methodology could be implemented in other wind turbine components to estimate the fatigue damage as the tower or the jacket. The estimation of the 10 minutes fatigue damage could be improved by increasing the length of the aero-elastic simulation and adding changes in turbulence intensity and shear exponent, however, this also will increase the computational cost when transferring the loads to the 2D shell blade model or by taking into consideration more uncertainty due to the turbulence intensity, wind direction, and shear exponent. 


\section{REFERENCES}

[1] C. Bak, F. Zahle, R. Bitsche, T. Kim, A. Yde, L. C. Henriksen, M. H. Hansen, J. P. A. A. Blasques, M. Gaunaa, and A. Natarajan, "The dtu 10-mw reference wind turbine," in Danish Wind Power Research 2013, 2013.

[2] J. M. Jonkman and M. L. Buhl Jr, "Fast user's guide-updated august 2005," tech. rep., National Renewable Energy Lab.(NREL), Golden, CO (United States), 2005.

[3] G. Guideline and G. Lloyd, "Guideline for the certification of wind turbines," Hamburg: Germanischer Lloyd Wind Energie Gmb H, 2010.

[4] D. Caous, N. Lavauzelle, J. Valette, and J.-C. Wahl, "Load application method for shell finite element model of wind turbine blade," Wind Engineering, 2018.

[5] N. K. Dimitrov, "Structural reliability of wind turbine blades: Design methods and evaluation," 2013.

[6] Z. Hashin, "Fatigue failure criteria for unidirectional fiber composites," 1981.

[7] C. L. Bottasso, F. Campagnolo, A. Croce, S. Dilli, F. Gualdoni, and M. B. Nielsen, "Structural optimization of wind turbine rotor blades by multilevel sectional/multibody/3d-fem analysis," Multibody System Dynamics, vol. 32, no. 1, pp. 87-116, 2014.

[8] W. Hu, K. Choi, and H. Cho, "Reliability-based design optimization of wind turbine blades for fatigue life under dynamic wind load uncertainty," Structural and Multidisciplinary Optimization, vol. 54, no. 4, pp. 953-970, 2016.

[9] Y. Liu and S. Mahadevan, "A unified multiaxial fatigue damage model for isotropic and anisotropic materials," International Journal of Fatigue, vol. 29, no. 2, pp. 347-359, 2007.

[10] L. Zhao, K. Choi, and I. Lee, "Metamodeling method using dynamic kriging for design optimization," AIAA journal, vol. 49, no. 9, pp. 2034-2046, 2011.

[11] R. M. Slot, J. D. Sørensen, B. Sudret, L. Svenningsen, and M. L. Thøgersen, "Surrogate model uncertainty in wind turbine reliability assessment," Renewable Energy, vol. 151, pp. 1150-1162, 2020.

[12] M. Miner, “Cumulative damage in fatigue," Journal of Applied Mechanics, vol. 12, no. 3, pp. 159$164,1945$.

[13] T. J. Santner, B. J. Williams, W. Notz, and B. J. Williams, The design and analysis of computer experiments, vol. 1. Springer, 2003.

[14] B. Sudret, "Polynomial chaos expansions and stochastic finite element methods," Risk and reliability in geotechnical engineering, pp. 265-300, 2014.

[15] Engie, "La haute borne data (2017-2020)." https://opendata-renewables.engie.com/explore/index, 2019. Accessed October 22, 2019.

[16] J. S. Touma, "Dependence of the wind profile power law on stability for various locations," Journal of the Air Pollution Control Association, vol. 27, no. 9, pp. 863-866, 1977.

[17] M. Kaltschmitt, W. Streicher, and A. Wiese, Renewable energy: technology, economics and envi- 
ronment. Springer Science \& Business Media, 2007.

[18] N. Dimitrov, A. Natarajan, and M. Kelly, "Model of wind shear conditional on turbulence and its impact on wind turbine loads," Wind Energy, vol. 18, no. 11, pp. 1917-1931, 2015.

[19] D. Standard et al., "Wind turbines-part 1: Design requirements," IEC61400-1, Copenhagen, 2005.

[20] B. J. Jonkman and M. L. Buhl Jr, “Turbsim user's guide,” tech. rep., National Renewable Energy Lab.(NREL), Golden, CO (United States), 2006.

[21] G. B. Arfken and H. J. Weber, "Mathematical methods for physicists," 1999.

[22] E. W. Weisstein, "Discrete fourier transform," https://mathworld. wolfram. com/, 2002.

[23] J. Mandell and D. Samborsky, "Snl/msu/doe composite material fatigue database mechanical properties of composite materials for wind turbine blades version 25.0," Montana State University, Bozeman, 2016.

[24] W. Hu, K. Choi, O. Zhupanska, and J. H. Buchholz, "Integrating variable wind load, aerodynamic, and structural analyses towards accurate fatigue life prediction in composite wind turbine blades," Structural and Multidisciplinary Optimization, vol. 53, no. 3, pp. 375-394, 2016.

[25] A. E. ASTM, "1049-standard practices for cycle counting in fatigue analysis," West Conshohocken (PA), 2003.

[26] R. M. Jones, Mechanics of composite materials. CRC press, 1998.

[27] Y. Liu and S. Mahadevan, "Probabilistic fatigue life prediction of multidirectional composite laminates," Composite Structures, vol. 69, no. 1, pp. 11-19, 2005.

[28] S. Sarkani, G. Michaelov, D. P. Kihl, and D. L. Bonanni, "Comparative study of nonlinear damage accumulation models in stochastic fatigue of frp laminates," Journal of Structural Engineering, vol. 127, no. 3, pp. 314-322, 2001.

[29] A. Wöhler, Über die festigkeitsversuche mit eisen und stahl. Ernst \& Korn, 1870.

[30] A. E73910, "Standard practice for statistical analysis of linear or linearized stress-life (sn) and strain-life ( $\varepsilon$-n) fatigue data," 2015.

[31] A. Vassilopoulos and R. Nijssen, "Fatigue life prediction of composite materials under realistic loading conditions (variable amplitude loading)," in Fatigue life prediction of composites and composite structures, pp. 293-333, Elsevier, 2010.

[32] A. P. Vassilopoulos, B. D. Manshadi, and T. Keller, "Influence of the constant life diagram formulation on the fatigue life prediction of composite materials," International journal of fatigue, vol. 32, no. 4, pp. 659-669, 2010.

[33] A. Géron, Hands-on machine learning with Scikit-Learn, Keras, and TensorFlow: Concepts, tools, and techniques to build intelligent systems. O’Reilly Media, 2019.

[34] S. Seabold and J. Perktold, "statsmodels: Econometric and statistical modeling with python," in 9th Python in Science Conference, 2010.

[35] L. Devroye, "Nonuniform random variate generation," Handbooks in operations research and management science, vol. 13, pp. 83-121, 2006. 